\title{
Margin Involved by Invasive Melanoma
}

National Cancer Institute

\section{Source}

National Cancer Institute. Margin Involved by Invasive Melanoma. NCI Thesaurus. Code C159232.

A result that the marg in is involved by invasive melanoma. 